\title{
Post-Error Slowing as a Consequence of Disturbed Low-Frequency Oscillatory Phase Entrainment
}

\author{
Ruud L. van den Brink, ${ }^{1,2}$ Syanah C. Wynn, ${ }^{1}$ and Sander Nieuwenhuis ${ }^{1,2}$ \\ ${ }^{1}$ Institute of Psychology, Leiden University, 2333AK Leiden, The Netherlands, and ${ }^{2}$ Leiden Institute for Brain and Cognition, 2333AK Leiden, The \\ Netherlands
}

\begin{abstract}
A common finding across many reaction time tasks is that people slow down on trials following errors, a phenomenon known as post-error slowing. In the present study, we tested a novel hypothesis about the neural mechanism underlying post-error slowing. Recent research has shown that when task-relevant stimuli occur in a rhythmic stream, neuronal oscillations entrain to the task structure, thereby enhancing reaction speed. We hypothesized that under such circumstances post-error slowing results from an error-induced disturbance of this endogenous brain rhythm. To test this hypothesis, we measured oscillatory EEG dynamics while human subjects performed a demanding discrimination task under time pressure. We found that low-frequency neuronal oscillations entrained to the stimulus presentation rhythm, and that the low-frequency phase at stimulus onset predicted the speed of responding. Importantly, we found that this entrainment was disrupted following errors, and that the degree of phase disturbance was closely related to the degree of post-error slowing on the subsequent trial. These results describe a new mechanism underlying behavioral changes following errors.
\end{abstract}

Key words: EEG; entrainment; errors; low-frequency; oscillations; post-error slowing

\section{Introduction}

One of the most common findings in empirical studies using reaction time (RT) measures is that RTs slow down on trials following errors, a phenomenon known as post-error slowing (PES; Rabbitt, 1966; Laming, 1979). PES has been shown to occur across many different tasks and response modalities, including go/no-go (Cohen et al., 2009); flanker (Cavanagh et al., 2009; Eichele et al., 2010); categorization (Dudschig and Jentzsch, 2009; Jentzsch and Dudschig, 2009); Stroop (Gehring and Fenscik, 2001); Simon (Ridderinkhof, 2002); and saccade countermanding tasks (Endrass et al., 2005). Researchers have proposed several accounts of the cognitive mechanisms that are responsible for PES (Danielmeier and Ullsperger, 2011), suggesting that PES can arise from strategic adjustments in response caution (Botvinick et al., 2001; Dutilh et al., 2012a) as well as detrimental processing interference caused by the error (Jentzsch and Dudschig, 2009; Notebaert et al., 2009). However, although studies have started to elucidate the neural mechanisms underlying errorrelated strategic adjustments (King et al., 2010; Danielmeier et al., 2011; Narayanan et al., 2013), the neural basis of error-related processing interference is still poorly understood.

\footnotetext{
Received Nov. 27, 2013; revised June 18, 2014; accepted July 5, 2014.

Author contributions: R.L.v.d.B., S.C.W., and S.N. designed research; R.L.v.d.B., S.C.W., and S.N. performed research; R.L.v.d.B. analyzed data; R.L.v.d.B., S.C.W., and S.N. wrote the paper.

This research was supported by a Starting Independent Researcher Grant of the European Research Council. We thank Dr. Tobias Donner and Dr. Michael X. Cohen for their helpful comments.

The authors declare no competing financial interests.

Correspondence should be addressed to Ruud L. van den Brink, Wassenaarseweg 52, 2333AK Leiden, The Netherlands. E-mail: r.l.van.den.brink@fsw.leidenuniv.nl.

DOI:10.1523/JNEUROSCI.4991-13.2014

Copyright $\odot 2014$ the authors $\quad 0270-6474 / 14 / 3411096-10 \$ 15.00 / 0$
}

In the present study, we tested the hypothesis that this component of PES results from an error-evoked disturbance of internally generated brain rhythmicity. It has been suggested that under circumstances of high external predictability, the timing of endogenous periodic processes is systematically aligned with the environmental rhythmicity (Large and Jones, 1999; Schroeder and Lakatos, 2009). Such temporal alignment can be implemented by neuronal oscillations, which are rhythmic fluctuations in the excitability of large-scale neuronal ensembles (Wang, 2010). This oscillatory phase locking, or entrainment, ensures that behaviorally relevant stimuli are processed in the optimal neuronal context, thereby optimizing the speed and accuracy of behavioral responses. Indeed, low-frequency neuronal oscillations have been shown to align with stimulus presentation rates, are modulated by expectations about stimulus onset, and predict the latency of behavioral responses (Lakatos et al., 2008; Schroeder and Lakatos, 2009; Saleh et al., 2010; Stefanics et al., 2010; Besle et al., 2011; Henry and Obleser, 2012).

To test our hypothesis that errors slow down subsequent responses through a disturbance of low-frequency EEG oscillatory phase entrainment, we examined the oscillatory dynamics of EEG signals from participants performing a modified Eriksen flanker task under high time pressure. We found that low-frequency neuronal oscillations entrained to the stimulus presentation rhythm, and that the low-frequency phase at stimulus onset predicted the speed of response. Importantly, we found that this entrainment was disrupted following errors, and that the degree of phase disturbance was closely related to the degree of PES on the subsequent trial. These results support our novel hypothesis regarding the neural origin of PES. 


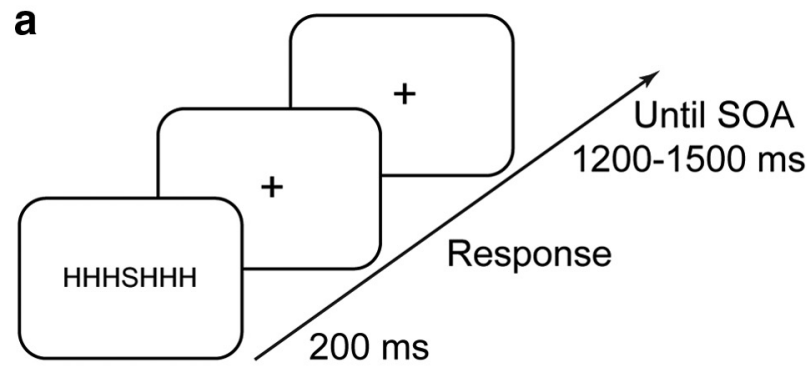

C

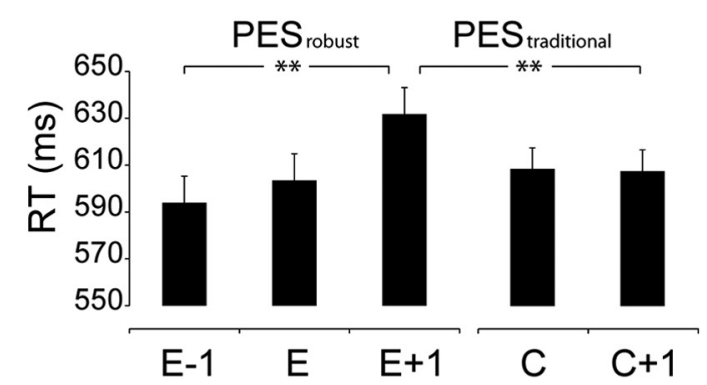

e

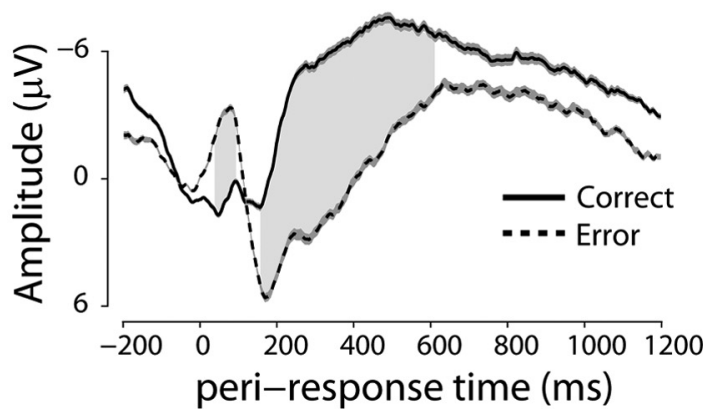

b

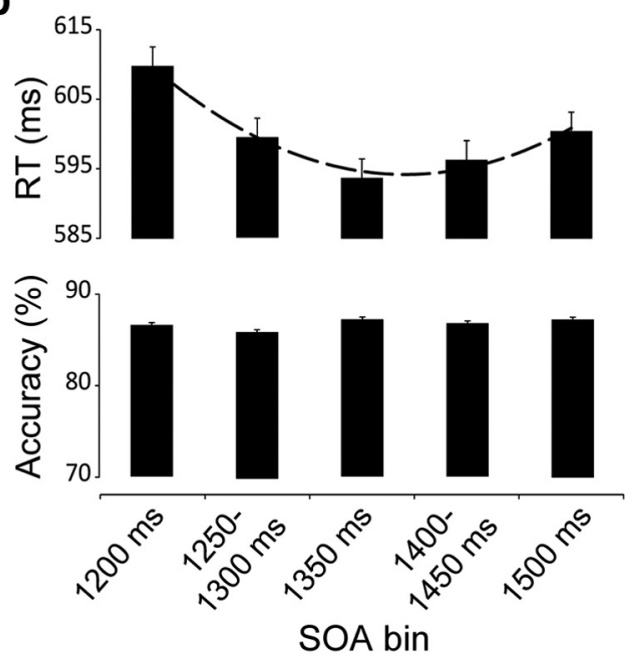

d

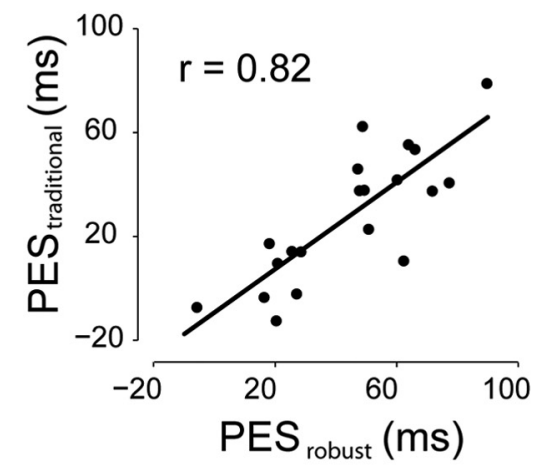

Figure 1. Task, behavioral, and ERP results. $\boldsymbol{a}$, Timing of trial events. $\boldsymbol{b}$, RT and accuracy as a function of (binned) preceding SOA. Intermediate bins are averaged for display purposes. The dashed line in the top is a fitted quadratic curve. $c$, Behavioral results showing post-error slowing, as measured by both the traditional and robust methods of quantifying PES. Asterisks indicate significant $(p<0.001)$ differences. $\boldsymbol{d}, \mathrm{PES}_{\text {robust }}$ and PES traditional correlated significantly across subjects $(p<0.01)$. $\boldsymbol{e}$, Response-locked event-related potentials for correct and error trials, averaged across electrodes $F z$ and $C z$. Shaded area indicates a significant $(p<0.001)$ difference between conditions. All error bars show the SEM.

\section{Materials and Methods}

Participants. Twenty-one participants (age range, 17-29 years; 17 female, all right handed) gave informed consent to take part in this study. Following EEG artifact rejection, one participant was excluded from further analysis due to an insufficient number of error trials $(<30)$. All participants had normal or corrected-to-normal vision, and were free from any neurological or psychiatric disorders. Participants were recruited via the Leiden University Research Participation website and received $€ 7.50 / \mathrm{h}$ or course credit in compensation. The experiment was approved by the Leiden University Institute of Psychology Ethics Committee.

Behavioral task. The participants performed a modified version of the flanker task (Eriksen and Eriksen, 1974), in which a target letter ("H," "K," "C," or "S") was flanked by three identical flanker letters (" $\mathrm{H}$, , "K," "C," or "S") on each side. Participants had to classify the target letter by giving one of two left-hand responses or one of two right-hand responses. The flanking letters were always incongruent with the target letter (e.g., SSSHSSS or KKKCKKK) and mapped to a finger of the hand opposite to the hand associated with the correct response. This ensured similar difficulty across trials, enabling cross-trial comparisons of RTs, while retaining a sufficiently high error rate.

Stimuli were presented in black on a white background for $200 \mathrm{~ms}$ at $2.77^{\circ}$ horizontal visual angle and at a viewing distance of $120 \mathrm{~cm}$. Stimulus onset asynchronies (SOAs) were randomly selected from a uniform distribution with a mean of $1350 \mathrm{~ms}$, and varying between 1200 and 1500 ms with 50 ms increments (Fig. 1a). During the intertrial interval, a black fixation cross was shown. Participants were instructed to keep their eyes fixated on the cross at all times. Between task blocks, participants received RT and accuracy feedback, and were pressed for speed. In total, there were 10 blocks of 104 trials each. Participants practiced the task beforehand (120 trials) to ensure that they understood the task.

Because several studies have found that PES is exclusively found for errors of which participants are aware (Hughes and Yeung, 2011; Murphy et al., 2012), we conducted a pilot experiment $(N=4)$ to verify whether participants detected the errors made in our challenging version of the flanker task. If they detected an error, participants were to press an error-signaling button (space bar) with their thumb, immediately after the error and before the next trial started (an additional task on top of the already demanding flanker task). All other methods were identical to those described for the main experiment. Participants correctly signaled $>76 \%$ of their errors, which provides a lower bound on the percentage of aware errors, while misclassifications of correct responses as errors were rare $(<2 \%)$. This suggests that participants were aware of the large majority of their errors.

Behavioral data acquisition and analysis. The experiment was run on a personal computer with an 18 inch monitor. Stimulus presentation and the recording of responses were performed using E-Prime, version 2.0 (Psychology Software Tools). 
To quantify PES, researchers usually subtract the mean RT (MRT) on post-error trials from the MRT on post-correct trials MRT $_{\text {post-error }}-$ $\mathrm{MRT}_{\text {post-correct }}$ ). This will hereafter be referred to as $\mathrm{PES}_{\text {traditional }}$ (Dutilh et al., 2012b). However, Dutilh et al. (2012b) have shown that this measure of PES is vulnerable to confounds related to global performance fluctuations. They therefore proposed an alternative measure of PES, dubbed $\mathrm{PES}_{\text {robust }}$. Following this method, we conducted a pairwise comparison of correct trials around each error $\left(\mathrm{RT}_{\text {post-error }}-\mathrm{RT}_{\text {pre-error }}\right)$, resulting in single-trial values of PES. For the calculation of $\mathrm{PES}_{\text {robust }}$, we included error trials that were both preceded and followed by at least one correct trial. To test for group-level significance of $\mathrm{PES}_{\text {traditional }}$ and $\mathrm{PES}_{\text {robust }}$, MRTs on post-error trials were compared with postcorrect and pre-error trials, respectively, with one-tailed pairedsample $t$ tests. Additionally, Pearson's correlation was used to determine whether PES traditional $_{\text {and }}$ PES robust $_{\text {were correlated. }}$ wers

EEG recording and preprocessing. EEG data were recorded using a BioSemi ActiveTwo system from the following 18 electrodes placed according to the international 10/20 system: F3; Fz; F4; C3; Cz; C4; P3; Pz; P4; PO7; PO3; POz; PO4; PO8; O1; Oz; $\mathrm{O}_{2}$; and Iz. Additionally, a reference electrode was placed on each earlobe, and bipolar electro-oculogram (EOG) recordings were obtained from electrodes placed $\sim 1 \mathrm{~cm}$ lateral of the outer canthi (horizontal EOG), and from electrodes placed $\sim 1 \mathrm{~cm}$ above and below the left eye (vertical EOG). During acquisition, impedances were kept $<30 \mathrm{k} \Omega$. The EEG signal was preamplified at the electrode to improve the signal-to-noise ratio with a gain of $16 \times$, and digitized at 24-bit resolution with a sampling rate of $1024 \mathrm{~Hz}$. Each active electrode was measured on-line with respect to a common mode sense active electrode producing a monopolar (nondifferential) channel.

All EEG data were analyzed in MATLAB 2011b, using the EEGLAB toolbox (Delorme and Makeig, 2004) and custom in-house code. First, EEG data were downsampled to $512 \mathrm{~Hz}$ and rereferenced off-line to the average of the earlobe electrodes. Next, to remove drifts, the continuous EEG data were high-pass filtered off-line at $0.5 \mathrm{~Hz}$ with a zero phase-shift, two-way, least-squares, finite impulse response filter (constructed using the MATLAB firl function, which smooths the filter kernel using a Hamming window by default). Zero phase-shift filters prevent the introduction of filter artifacts that could distort real oscillatory phase information. Filtering the continuous time course rather than epoched data also prevented edge artifacts from contaminating the data. Nevertheless, to verify that high-pass filtering did not influence low-frequency phase estimates, we also ran the relevant analyses on data to which no high-pass filter was applied, and found that it did not influence the results.

Additionally, the EEG data were notch filtered at $50 \mathrm{~Hz}$ to remove line noise. Following filtering, the continuous data were segmented into epochs ranging from -1 to 3 s centered on stimulus onset and baseline corrected by subtracting the average offset during the -400 to $-100 \mathrm{~ms}$ prestimulus window. Next, the following types of trials were excluded from further analysis: trials in which the participant failed to respond: trials that were part of a sequence of more than three consecutive errors; and trials with an RT $>1200$ ms. Trials with transient artifacts and eye movements were manually rejected. Following trial rejection, on average 609 correct trials per participant and 78 error trials remained (393 and 47 respective lower limits), and 693 post-correct trials and 94 post-error trials remained (501 and 51 respective lower limits). Next, eye blinks and continuous electromyogram artifacts were identified using JADE independent component analysis as implemented in the EEGLAB toolbox (Delorme and Makeig, 2004), and the corresponding components were removed from the data.

Event-related potential analysis. To confirm that our task showed the event-related potential (ERP) components that are typically found during flanker tasks-the error-related negativity and error positivity-we computed response-locked ERPs for correct and error trials, and baseline corrected them by subtracting the average offset during the -100 to $0 \mathrm{~ms}$ preresponse window. These ERPs were then compared on each time point with two-tailed $t$ tests with a $p$ value threshold of $\leq 0.001$ and cluster corrected for multiple comparisons across time points (Maris and Oostenveld, 2007).

Time-frequency analyses. Spectral power and phase dynamics were extracted via Morlet wavelet decomposition. Wavelet decomposition in- volves convolving the data with a set of Gaussian-windowed complex sine waves, defined here as follows:

$$
\psi_{f_{w}}=e^{2 i \pi f_{w} t} \cdot e^{\frac{-t^{2}}{2 s_{w}^{2}}}
$$

where $f_{w}$ denotes frequency, which ranged from 0.5 to $30 \mathrm{~Hz}$ with 30 logarithmically spaced steps; $t$ denotes time, and $s_{w}$ determines the width of the Gaussian window, and thus the tradeoff between time and frequency precision. A wider Gaussian will result in a wider wavelet, and thus results in more temporal smearing of instantaneous power/phase estimates. Because we were primarily interested in lower frequencies, and the lower frequencies inherently span a wider temporal range, we preferred to minimize temporal smearing at the expense of frequency resolution in the lower frequency bands. We therefore linearly increased the Gaussian width with frequency, as follows:

$$
s_{w}=\frac{c_{w}}{2 \pi f_{w}},
$$

where $c_{w}$ denotes the number of wavelet cycles, which ranged from 3 to 12 .

To accommodate the large wavelet width at the lower frequencies, before running wavelet decomposition we expanded each data epoch with a mirror (time-reversed) image of itself such that each epoch consisted of a mirror image of the epoch, then the "true" epoch, and another mirror image following it. This increased the effective epoch size from 4 to $12 \mathrm{~s}$ while preserving data continuity, thereby preventing the introduction of transients in the signal that could cause edge artifacts (Cohen, 2014).

After convolution, frequency-specific instantaneous power $(P)$ is given by the following:

$$
P\left(f_{w}, t\right)=\operatorname{ReM}\left(f_{w}, t\right)^{2}+i M\left(f_{w}, t\right)^{2},
$$

where $R e M$ and $i M$ denote the magnitude of the real and imaginary components of the convolution result, respectively. To enable comparisons across frequency bands, power was converted to decibel scale via the following: $10 \times \log 10\left[P(t, f) / P\left(t_{\text {baseline }}, f\right)\right]$, where $t_{\text {baseline }}$ baseline ranged from -300 to $-100 \mathrm{~ms}$. Frequency-specific instantaneous phase $(\varphi)$ is given by the angle of the convolution result (arctangent of $i M$ over ReM) at time $t$.

To demonstrate the entrainment of EEG oscillations to the task rhythm, we computed intertrial phase coherence (ITPC), a measure of the consistency of phase across trials. This measure ranges between 0 (no consistency) and 1 (perfect consistency), and can be computed using the following:

$$
\operatorname{ITPC}\left(f_{w}, t\right)=\left|n^{-1} \sum_{N=1}^{n} e^{i \phi\left(f_{w}, t\right)}\right|
$$

where $n$ is the number of trials.

Statistical analyses of time-frequency data. As randomly distributed phases across trials produce a known (Rayleigh) distribution, the significance of observed ITPC can be assessed by comparing it to ITPC under the null hypothesis. Conversely, a critical value $\left(\right.$ ITPC $\left._{\text {crit }}\right)$ at which ITPC significantly deviates from randomly distributed phase values can be calculated, given a $p$ value ( 0.001 in our case), via the following:

$$
\mathrm{ITPC}_{\text {crit }}=\sqrt{-\log (p) \cdot \mathrm{n}^{-1}}
$$

where $n$ is the number of trials (Zar, 1999; Cohen, 2014). We thus calculated ITPC $_{\text {crit }}$, and for each time-frequency point values higher than the ITPC $_{\text {crit }}$ were considered to be significant.

We used nonparametric permutation testing to assess whether power deviated from the -300 to $-100 \mathrm{~ms}$ prestimulus baseline window, for each time-frequency point. Permutation testing is robust against violations of assumptions about data distributions that can occur with conventional parametric statistics (Maris and Oostenveld, 2007). First, within each frequency, the assignment of evoked power to "baseline" or "power" distributions were shuffled, and $t$ statistics specific to each time- 
frequency point were computed iteratively 1000 times. The resulting $t$ value distribution was then $z$ scored, and the standardized value of the nonshuffled comparison (actual baseline vs power) was then computed per time-frequency point. This procedure generated a time-frequency matrix with a $z$-value for each time-frequency point that indicated the statistical likelihood of finding the experimentally obtained power values, given that the null hypothesis (no difference in power compared with baseline) is true. To correct for multiple comparisons, the threshold for the time-frequency matrix was set as $z$-scores corresponding to $p$ values of $\leq 0.001$. In a second step, a distribution of maximum cluster sizes (the number of contiguous significant time-frequency points) under the null hypothesis was computed. The cluster size corresponding to the 95th percentile of this distribution was then taken as the lower bound for cluster correction of the time-frequency matrix, resulting in a clusterlevel threshold of $p<0.05$ (Cohen et al., 2012).

To test whether phase distributions differed from a uniform distribution, we used Rayleigh's test for uniformity (Fisher, 1993).

Fixed-effects analysis. Circular-linear correlations (i.e., correlations between single-trial phase and behavioral measures) were performed using a nonparametric permutation testing approach that was similar to the one described above. Correlation distributions under the null hypothesis in this case were created by shuffling the assignment of pooled behavioral data (i.e., RT or PES $_{\text {robust }}$ ) to phase information at the singletrial level. The test statistic, which was subsequently $z$ scored for each time-frequency point, was the correlation coefficient resulting from circular-to-linear correlation as implemented in the circular statistics (CircStat) toolbox (Berens, 2009).

To assess differences in average phase angle between conditions (i.e., correct vs error; post-correct vs post-error; and post-correct vs large and small PES bins), we again used a similar procedure, but instead shuffled the assignment of pooled single-trial phase to trial types to compute a standardized distribution of angle differences under the null hypothesis. We used the Watson-Williams test for angular means to obtain the teststatistic that was informative of the angle differences between conditions. This test statistic was subsequently standardized, similar to the analyses described above. Because there were more correct and post-correct trials than error and post-error trials, respectively, we matched these trial numbers between conditions by selecting a random subset of trials from the larger condition before computing differences between them in the average phase angle.

Random-effects analysis. Because the fixed-effects procedure described above does not take into account the between-subjects variance in effect size, we repeated these analyses for a number of select time-frequency points (stimulus onset, and $600 \mathrm{~ms}$ poststimulus/response), but without assuming constant between-subject variance. Specifically, we computed phase-RT and phase-PES correlations for each individual participant to obtain a distribution of correlation coefficients. Because circular-tolinear correlations are bound between 0 and 1 , correlation coefficients under the null hypothesis are unlikely to be exactly 0 . We therefore computed the correlations again but with shuffled phase behavior assignments for 1000 permutations. This resulted in a distribution of correlation coefficients under the null hypothesis. To assess the group-level significance of the observed distribution of correlation coefficients, we used a paired-sample $t$ test to compare them to the distribution under the null hypothesis averaged across permutations.

To test for differences in phase angle between conditions, we first computed the average phase angle and resultant vector length across trials for each participant and each condition. Then, group-level differences in phase angle between conditions were assessed using the parametric Hotelling paired-sample test for angular means (Zar, 1999). The extension of this test described by Zar (1999) takes into account the resultant vector lengths of the phase distributions of individual cases (participants, in our case), which carries meaningful information regarding the consistency of the phase angle difference between the average distributions. It is therefore more suited to be used in second-level, random-effects analyses than the Watson-Williams test.

\section{Results}

\section{Behavioral and ERP results}

The average RT on correct trials was $608 \mathrm{~ms}$ (SD, $69 \mathrm{~ms}$ ); the average RT on error trials was $604 \mathrm{~ms}$ (SD, $87 \mathrm{~ms} ; p=0.53)$. The average percentage of correct responses was $86.7 \%$ (SD, 5.8\%). To confirm that participants were sensitive to the average stimulus presentation rate (one stimulus every $1350 \mathrm{~ms}$ ), we binned correct trials by the preceding SOA and expected the shortest RTs in the $1350 \mathrm{~ms}$ bin. A repeated-measures ANOVA revealed that a correct RT had an inverted U-shaped relationship with the preceding SOA (Fig. $1 b$; quadratic trend: $F_{(1,19)}=12.73, p<0.01$ ), indicating that participants were good at estimating the average SOA of $1350 \mathrm{~ms}$. No such trend was found for accuracy $\left(F_{(1,19)}=\right.$ $0.8, p=0.38)$, ruling out the preceding SOA as a confounding factor in our comparisons between post-error and post-correct trials.

As a next step, we wanted to confirm that PES occurred in our flanker task with only incongruent stimuli. In line with our expectations, both $\mathrm{PES}_{\text {traditional }}$ (mean, $27 \mathrm{~ms}$; SD, $25 \mathrm{~ms}$ ) and $\mathrm{PES}_{\text {robust }}$ (mean, $44 \mathrm{~ms} ; \mathrm{SD}, 24 \mathrm{~ms}$ ) were significantly larger than 0 $(p<0.001$; Fig. $1 c)$. Although PES $_{\text {robust }}$ was significantly larger $(p<0.001)$, the two measures of PES were highly correlated $(p<$ 0.001 ; Fig. $1 d)$, suggesting that $\mathrm{PES}_{\text {robust }}$, although more precise (Dutilh et al., 2012b), does not provide radically different values for PES than the more traditional measure. Post-error accuracy (mean, 84.1\%; SD, 12.0\%) was somewhat lower than postcorrect accuracy (mean, $87.7 \%$; SD, $4.2 \%$ ), but this difference was not significant $(p=0.24)$. Figure $1 e$ shows that the errorrelated negativity and error positivity, two well known errorrelated ERP components, were present in our EEG data.

\section{Functional entrainment of oscillations}

To replicate previous results indicating that low-frequency oscillations entrain to the stimulus presentation rhythm (Lakatos et al., 2008; Schroeder and Lakatos, 2009; Stefanics et al., 2010; Henry and Obleser, 2012; Ng et al., 2012), we computed ITPC, a measure of the consistency of the oscillatory phase across trials. We calculated ITPC averaged across all trial types and electrodes to assess which frequencies showed the highest degree of consistency, and expected the greatest consistency in low $(<2 \mathrm{~Hz})$ frequencies, overlapping with our task rhythm. In line with this expectation, Figure $2 a$ shows that ITPC was significantly higher than expected by chance in the lowest frequency range. Additionally, the cluster of time-frequency points that showed significant ITPC extended into the higher frequencies.

To confirm the dependence of ITPC on the task rhythm, we ran a control experiment $(N=4)$ with the same task, except that the stimulus presentation rhythm was manipulated in a blockwise fashion $(0.5$ and $0.85 \mathrm{~Hz})$. All other task parameters were the same as in the main experiment. For each of the four participants, we found clearly dissociable peaks in ITPC (at 0.5 and $0.85 \mathrm{~Hz}$ ), which differed between blocks, indicating that oscillatory phase locking was highly dependent on the stimulus presentation rhythm.

Elevated ITPC can come about in two ways (Tallon-Baudry and Bertrand, 1999; Donner and Siegel, 2011). The first possibility is that ITPC arises due to cross-trial phase alignment of true, endogenously driven oscillatory activity. The second possibility is that ITPC is a result of stimulus-evoked activity, meaning that simple stimulus-evoked EEG components cause consistency in phase across trials. To exclude the possibility that stimulusevoked activity was the driving force behind consistency in phase across trials, we computed oscillatory power of stimulus-locked 

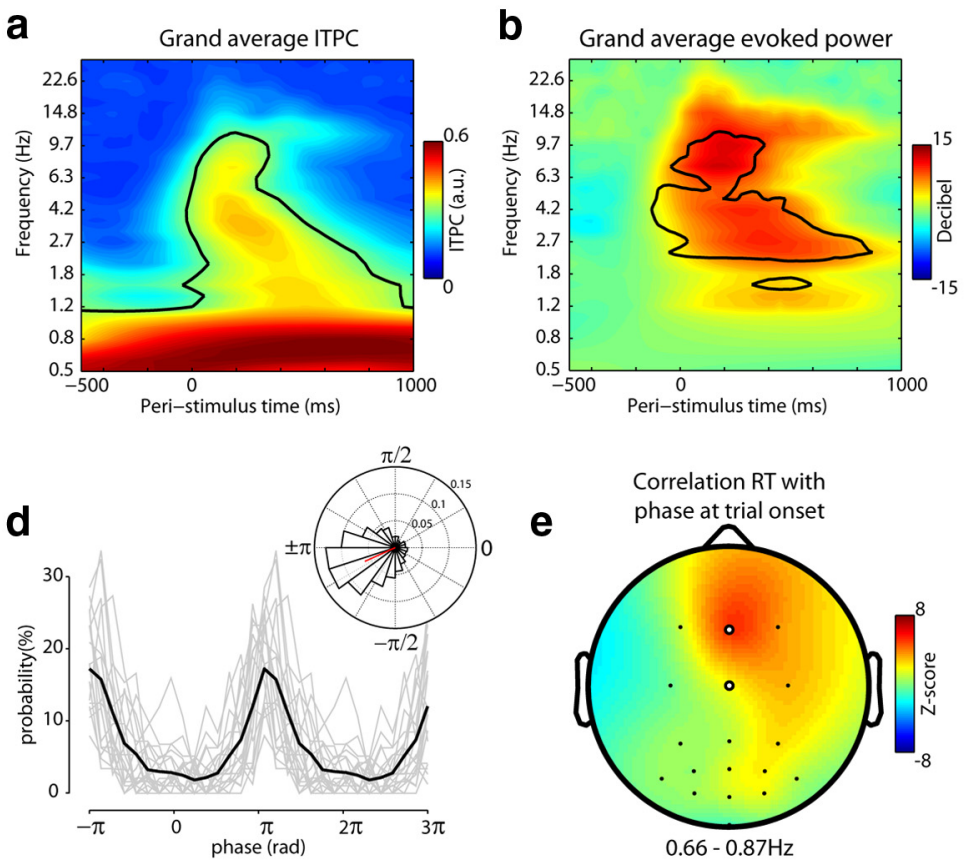

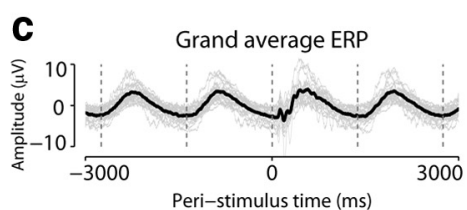

$f$
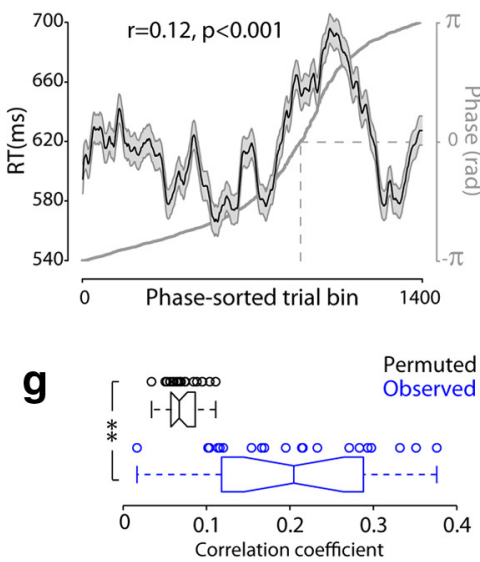

Figure 2. Functional entrainment of oscillations to the task rhythm. $\boldsymbol{a}$, Low-frequency oscillations entrain to the task rhythm, as indicated by ITPC averaged across all trials and all channels. $\boldsymbol{b}$, Evoked (ERP) power averaged across all conditions and all channels. Only frequencies above the task rhythm show significant increases in evoked power, suggesting that elevated low-frequency ITPC reflects the entrainment of endogenous oscillations. Significant $(p<0.001)$ regions are outlined in black using the MATLAB contourf function. $c$, Channel- and condition-averaged ERPs showing oscillations entrained to the stimulus presentation rhythm. The solid gray lines show the ERPs of individual participants. The solid black line shows the average. The vertical dashed gray lines show the time of average stimulus onsets. $\boldsymbol{d}$, Low-frequency $(0.76 \mathrm{~Hz})$ phase distribution at correct trial onset for the average of channels Fz and Cz. The solid black line shows the average. Gray lines show individual participants. The inset shows a rose histogram, where the radial extent of the bars indicates the probability of a given phase occurring on a single trial. The red line is the average vector of the histogram. $\boldsymbol{e}$, Topographical distribution of the $z$-scored correlation of correct RTs with low-frequency phase at trial onset. The two highlighted channels are Fz and C $z$, the average of which was used in all further reported analyses. $f$, Correct RTs sorted by the low-frequency $(0.76 \mathrm{~Hz})$ phase at trial onset, smoothed with a moving average of 100 trials for display purposes. The nonlinearity in the curve arises due to a correlation of phase with RT. The error bars represent the SEM and are the result of smoothing. The light gray line and corresponding $y$-axis on the right-hand side represent the phase for each of the sorted trials. $g$, Box plots of the correlation coefficients for permuted (black) and observed (blue) correlations between correct RT and $0.76 \mathrm{~Hz}$ phase at trial onset for the average of channels Fz and Cz. A 95\% confidence interval around the median of each distribution is indicated by the notches in the boxes. The whiskers extend to the most extreme values of each distribution. The circles show the correlation coefficients of individual participants. ${ }^{* *} p<0.001$.

trial-averaged (i.e., ERP) data. As Figure $2 b$ shows, no significant evoked power was found below $\sim 2 \mathrm{~Hz}$, indicating that ITPC in these low frequencies was likely driven by endogenous oscillatory activity. Above $\sim 2 \mathrm{~Hz}$, however, we found significant evoked power, indicating that elevated ITPC in those frequencies was most likely stimulus evoked. In sum, lower frequencies showed significant ITPC, which likely reflected entrainment of endogenous neuronal oscillations to the stimulus presentation rhythm, which is in line with our prediction. These entrained oscillations are readily visible in the stimulus-locked grand-average ERP (Fig. $2 c)$. Moreover, the phase angle of our frequency of interest $(0.76$ $\mathrm{Hz}$ ) at stimulus onset was highly consistent across participants (Fig. 2d; deviation from uniform distribution: Rayleigh's $z=$ $11.3, p<0.0001)$.

Next, to show that this entrainment is directly linked to task performance, we correlated correct RTs with single-trial instantaneous phase at trial onset, pooling the trials of all participants, and focusing on the frequency corresponding with the average stimulus presentation rate $(0.76 \mathrm{~Hz})$. The relationship between phase and RT was strongest over frontocentral scalp regions (Fig. $2 e$ ), in accord with previous findings (Stefanics et al., 2010; Henry and Obleser, 2012; Ng et al., 2012). Accordingly, all subsequent analyses were performed on the average of the signals recorded at $\mathrm{Fz}$ and $\mathrm{Cz}$. The significant phase-RT relationship $(r=0.12, p<$ 0.001 ) with trials pooled across participants is shown in more detail in Figure $2 f$. This correlation was also significant $(p<$ $0.001)$ at the group level with a random-effects analysis approach
(Fig. 2g). Thus, the entrainment of oscillations is functional, in that it predicts the latency of responding, and this effect is consistent across participants.

\section{Error-induced disturbance in phase entrainment}

To test our hypothesis that errors result in a disturbance of phase entrainment, we calculated the difference in phase angle between correct and error trials (response locked, i.e., aligned with the commission of errors/correct responses), and post-correct and post-error trials (stimulus locked, i.e., aligned with the onset of stimuli following errors/correct responses). If the average phase angle on correct responses facilitates response speed, then PES may reflect a temporary deviation from this phase angle. Note that if errors result in a systematic disturbance of phase entrainment, this will become apparent as a difference in phase angle following correct and erroneous responses rather than as a difference in phase-locking strength.

As Figure $3 a$ shows, following the response, the low-frequency phase angle on error trials significantly differs from that of correct trials. Although the difference in phase angle between correct and error trials is already evident before the response, this preresponse difference is likely the result of temporal smearing of the effect that is inherent to wavelet deconvolution. To confirm this, we reran our wavelet deconvolution with a lower number of wavelet cycles, thereby increasing the temporal resolution at the expense of frequency resolution. We found that this eliminated all preresponse differences in phase angle between errors and 

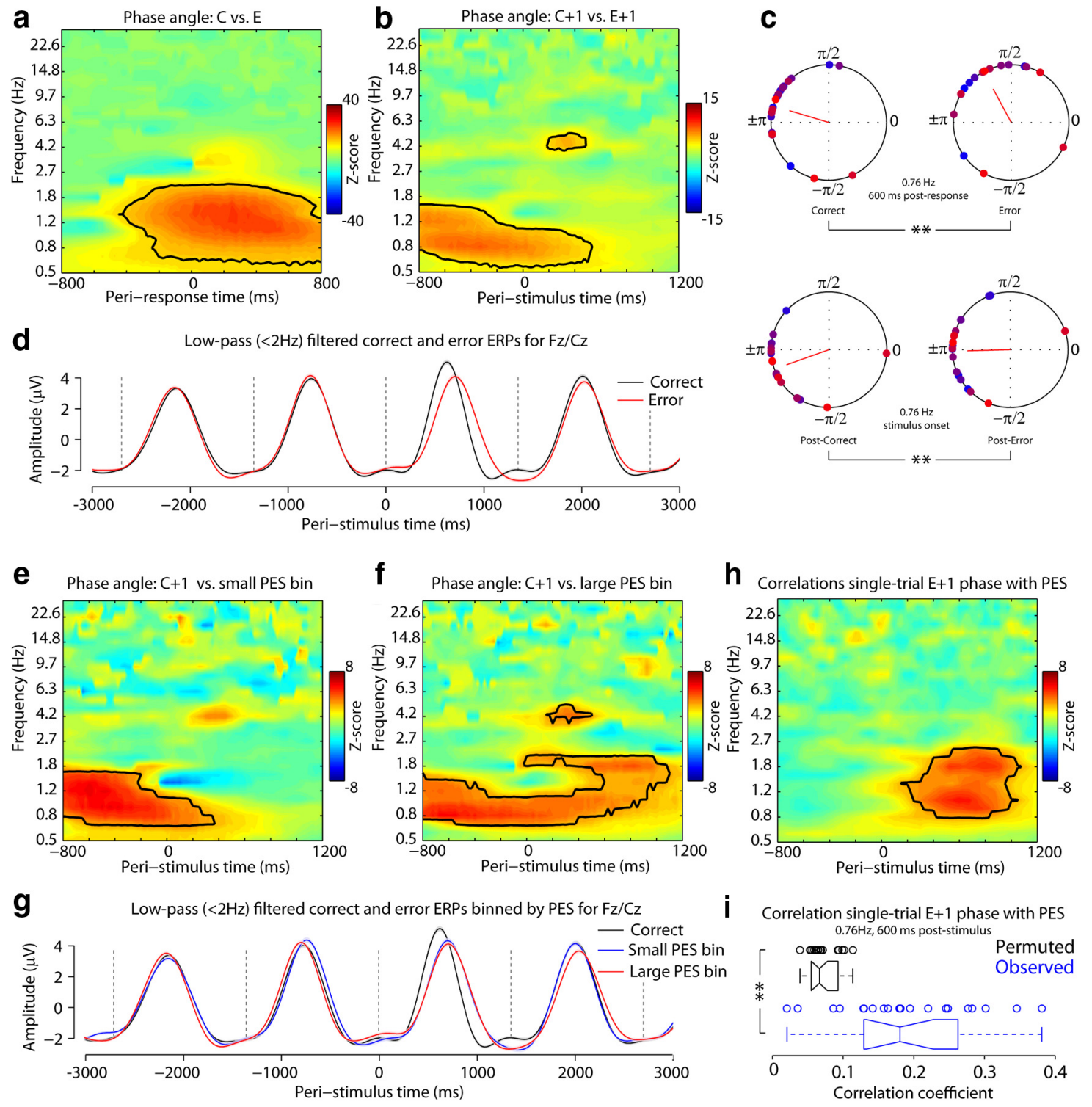

Correlation single-trial $\mathrm{E}+1$ phase with $\mathrm{PES}$

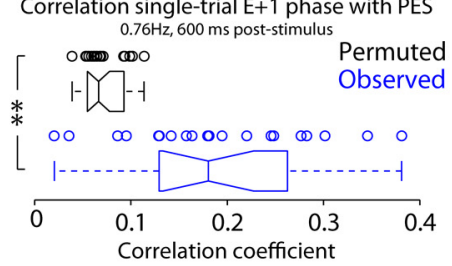

Figure 3. Relation between phase angle and post-error slowing. $\boldsymbol{a}$, The z-scored differences in absolute phase angle between correct (C) and error (E) trials, pooled across participants. Red colors indicate the phase on error trials significantly lagging behind correct trials. Time 0 indicates the time of a response. $\boldsymbol{b}$, The $z$-scored differences in absolute phase angle between post-correct $(\boldsymbol{C}+$ 1) and post-error $(E+1)$ trials, pooled across participants. Red colors indicate the phase on post-error trials significantly lagging behind post-correct trials. Time 0 indicates the onset of the stimulus. c, Low-frequency $(0.76 \mathrm{~Hz})$ phase distributions for post-correct and post-error trials for individual subjects. Each participant is denoted by a uniquely colored dot on the circle. The average vector of the distribution is shown in red. ${ }^{* *} p<0.001$. $d$, Low-pass $(<2 \mathrm{~Hz}$ )-filtered ERPs for the average of channels Fz and Cz. Error bars denote the SEM. Dashed vertical lines show the average stimulus onset times. $\boldsymbol{e}$, The $z$-scored phase angle differences between trials that show a low degree of PES (small PES bin) and post-correct trials. $\boldsymbol{f}$, The $z$-scored phase angle differences between trials that show a high degree of PES (large PES bin) and post-correct trials. $\boldsymbol{g}$, Same as $\boldsymbol{d}$, but error trials are binned according to PES robust $\boldsymbol{h}$, The $z$-scored correlations of single-trial phase on post-error trials

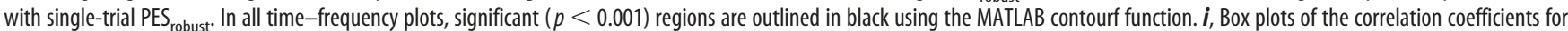
permuted (black) and observed (blue) correlations between single-trial $0.76 \mathrm{~Hz}$ phase at $600 \mathrm{~ms}$ post-stimulus on post-error trials and single-trial PES robust A $95 \%$ confidence interval around the median of each distribution is indicated by the notches in the boxes. The whiskers extend to the most extreme values of each distribution. The circles show the correlation coefficients of individual participants. ${ }^{* *} p<0.001$.

correct trials, while post-response differences were still present (results not shown). This indicates that the disturbance in phase entrainment following the response is likely to be error induced. The difference in phase angle following correct and error responses was highly consistent across participants (Fig. $3 c$, top; $\left.F_{(2,18)}=19.3, p<0.001\right)$. Figure $3 b$ shows that this error-induced disturbance in phase entrainment extends into the post-error trial, where it could potentially influence RT, thereby causing slowing on post-error trials. The difference in phase angle at trial onset for post-correct and post-error trials was highly consistent across participants (Fig. $3 c$, bottom; $F_{(2,18)}=6.04, p<0.001$ ).
The error-induced disturbance in entrainment is also visible in the low-pass filtered ERPs. Figure $3 d$ shows that around the average response time, the phase of the error-trial ERP starts to lag behind that of the correct-trial ERP. This phase difference extends into the subsequent trial and then dissolves again.

If the error-related disturbance in phase entrainment relates to how much PES occurs, phase angle should differ between trials that show a large degree of PES and trials with a small degree of PES. To test this prediction, we divided up the post-error trials into three equally sized bins according to the amount of $\mathrm{PES}_{\text {robust }}$, and compared, for each time and frequency point, the phase 
angle of these bins to the phase angle on post-correct trials. We expected to see a more pronounced disturbance in phase angle on trials with a large degree of PES. Consistent with our prediction, Figure $3, e$ and $f$, shows that the disturbance in phase entrainment extended for a longer period of time on trials with a large degree of PES. This indicates that the longer the disturbance in phase entrainment lasts following an erroneous response, the slower the participant is in responding on the subsequent trial. This strongly suggests a link between PES and disturbed phase entrainment.

The prolonged disturbance during trials that show a large degree of PES is also visible in the ERPs (Fig. $3 g$ ). Whereas the ERP of the small PES bin rejoins the ERP of correct trials before the trial $N+1$ response period, the ERP of the large PES bin is still out of phase with respect to correct trials during that period. The difference in $0.76 \mathrm{~Hz}$ phase angle between post-error trials with large PES and post-correct trials was highly consistent across participants (at stimulus onset: $F_{(2,18)}=7.32, p=0.0047$; at $600 \mathrm{~ms}$ post-stimulus: $\left.F_{(2,18)}=3.74, p=0.0053\right)$. The difference in phase angle between post-error trials with small PES and post-correct trials was not significant $\left(F_{(2,18)}=2.40, p=0.12\right)$, conceivably due to the fact that binning resulted in too few trials for an accurate estimate of average phase angle for each participant separately.

Next, to test directly whether PES can be predicted by the level of phase disturbance, we correlated phase on post-error trials with $\mathrm{PES}_{\text {robust }}$ at the single-trial level. Figure $3 h$ shows that on post-error trials, the low-frequency phase predicted PES, demonstrating a trial-by-trial relationship between disturbed phase entrainment and PES. Interestingly, the time window in which phase predicted PES overlapped with the period during which trials with large PES (Fig. 3f), but not trials with small PES (Fig. $3 e$ ), differed in phase angle from post-correct trials. This suggests that post-error trials were generally characterized by some degree of phase disturbance, and that the amount of PES on a given trial was mainly dependent on the duration of the error-induced phase disturbance. The correlation between post-error phase around the time of the response $(\sim 600 \mathrm{~ms})$ and single-trial PES was highly consistent across participants (Fig. 3i).

Finally, to confirm that the disturbance is specific to the entrained low-frequency oscillations rather than reflecting a broader spectral perturbation, we computed power and ITPC for correct and error trials, and post-correct and post-error trials, as well as respective differences between them. As can be seen in Figure 4, there are no significant differences in power or ITPC between conditions, except for the well documented errorrelated increase in theta-band power (Narayanan et al., 2013). This indicates that the phase angle differences between correct and error trials, and between post-correct and post-error trials are unlikely to result from a broad-band perturbation. Instead, the results are in line with our hypothesis of a specific phase disturbance in the task-entrained frequency.

\section{Discussion}

In the present study, we tested the hypothesis that PES results from a disturbance of internally generated brain rhythmicity. Together, our results show the following: (1) that endogenous low-frequency neuronal oscillations entrain to the stimulus presentation rhythm; (2) that the entrainment facilitates speeded responding; (3) that entrainment is disturbed following the commission of an error; and, importantly, (4) that the level of disturbance of entrainment predicts how much slowing occurs on the following trial. These results support our novel hypothesis about the neural origin of PES.
What might be the mechanism underlying this error-related disturbance of phase entrainment? One possibility is that errors evoke a transient process that temporarily distracts from the current task and therefore results in slower responding on the subsequent trial (Jentzsch and Dudschig, 2009). This possibility is suggested by the orienting account of PES (Notebaert et al., 2009), which posits that errors, due to their infrequent occurrence, automatically draw attention away from the ongoing task, much like other surprising events tend to do. The orienting account is supported by the finding that slowing occurs following correct trials instead of errors when correct responses are infrequent (Notebaert et al., 2009; Núñez Castellar et al., 2010); and by the observation that participants who make fewer errors (i.e., for whom errors are more unexpected) show larger PES (Steinborn et al., 2012). Our findings suggest that this hypothesized orienting response may cause the phase angle of entrained oscillations to deviate from the more advantageous phase angle observed following correct trials, thus leading to slower responding. This account is consistent with recent studies that have linked the phase of ongoing delta oscillations to the rate of perceptual evidence accumulation (Wyart et al., 2012; Cravo et al., 2013).

Other evidence in support of a limited duration orienting response during which attention is distracted from the task is provided by studies that examined the relationship between posterror performance, and the interval between the error and the subsequent trial (Dudschig and Jentzsch, 2009; Jentzsch and Dudschig, 2009; Danielmeier and Ullsperger, 2011). By varying the response stimulus interval (RSI), these studies found that short RSIs $(<500 \mathrm{~ms})$ are typically associated with large PES and a post-error decrease in accuracy. As the RSI increases to $\geq 1000$ $\mathrm{ms}$, PES strongly diminishes and the post-error decrease becomes a post-error increase in accuracy. We found an intermediate pattern of results (modest PES, slightly decreased accuracy after errors) that seems consistent with the intermediate length of our average RSI ( $\sim 750 \mathrm{~ms})$. The orienting account can explain these findings by claiming that at short RSIs attentional reorientation is the dominant cause for the impaired performance on the subsequent trial. As the RSI increases, it becomes increasingly likely that the system has recovered from the orienting response by the time the next stimulus is presented. The timing of the errorinduced disturbance in phase entrainment observed here, which partially overlapped with the subsequent trial (and more so on trials characterized by large PES), is consistent with the notion that at intermediate RSIs there is a substantial probability of overlap between the orienting response and the subsequent trial. Of course, due to the correlative nature of our methods, we cannot unequivocally conclude that disturbed entrainment is the causative mechanism underlying PES. This issue can be addressed by manipulating entrainment directly, for example, by using transcranial magnetic stimulation.

A disturbance in phase entrainment is unlikely to be the only cause of PES. Dutilh et al. (2012a), using drift diffusion modeling of performance on a lexical decision task, found that when the RSI is relatively long (1000 ms), PES can be attributed almost exclusively to increased response caution. This suggests that a strategic change in response threshold (Botvinick et al., 2001) can also contribute to PES. Another study using drift diffusion model analysis found that PES could be explained by a change in several model parameters, including an increased response threshold and a decreased rate of evidence accumulation, which is consistent with disturbed phase entrainment (White et al., 2010). However, in this study participants received trial-to-trial feedback, so post-error effects may have been contaminated by the possibly 
a

\section{a}
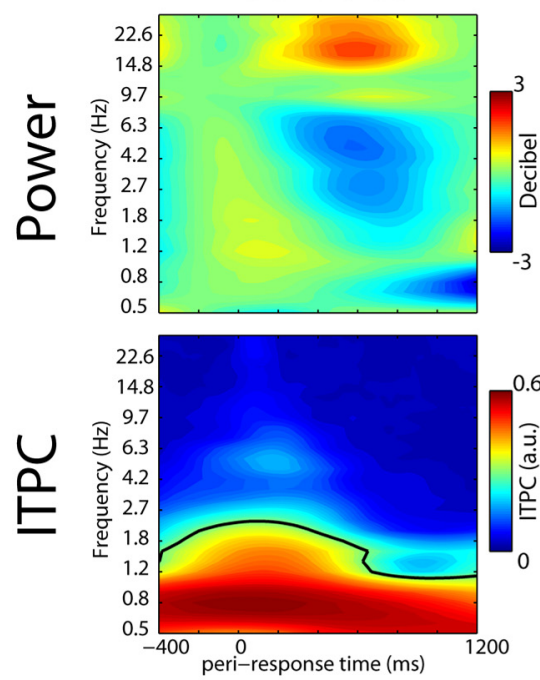

b
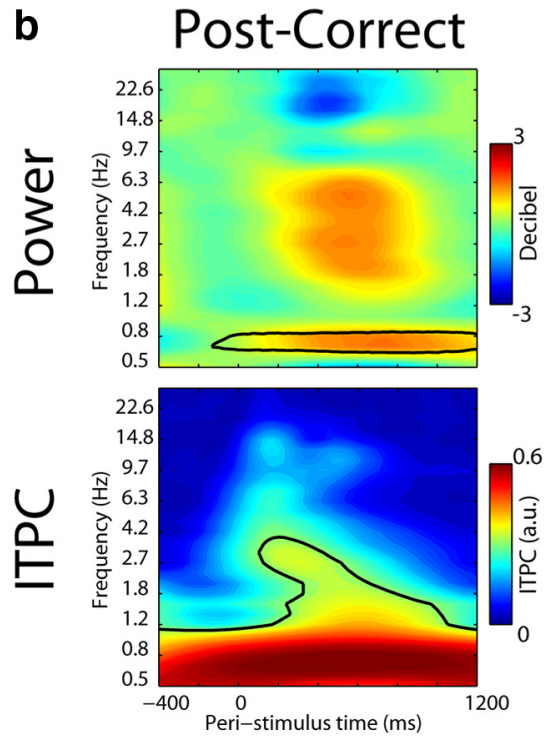

Error
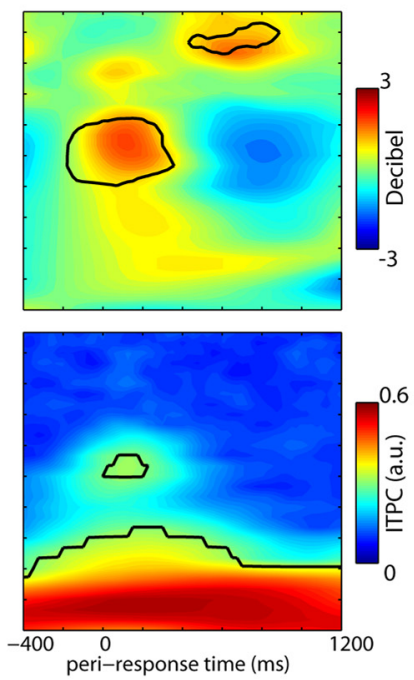

Post-Error
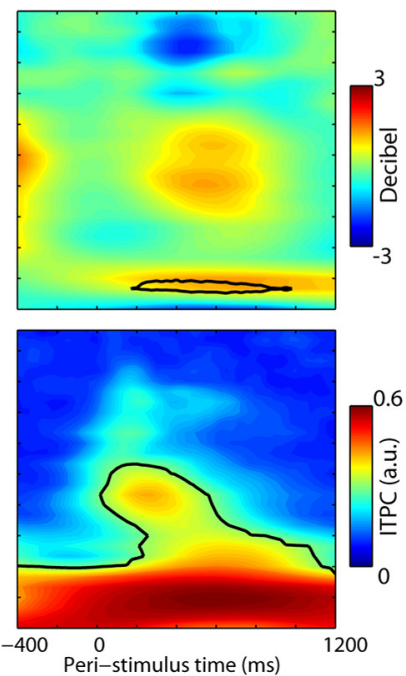

Difference
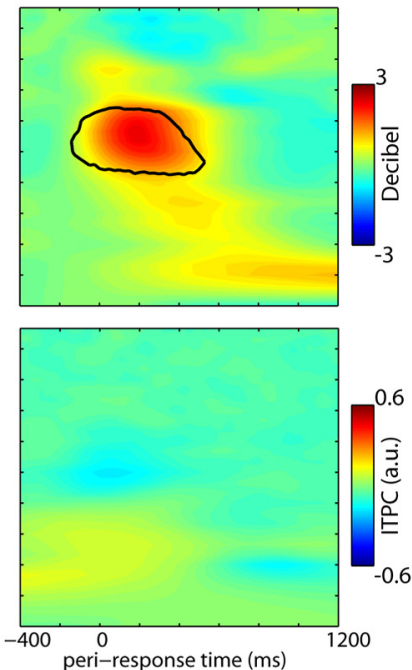

\section{Difference}
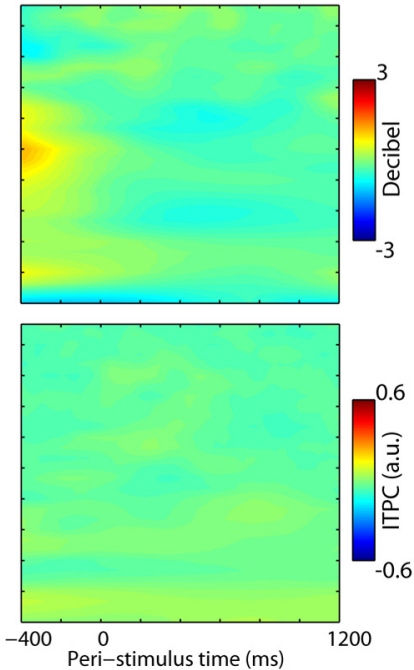

Figure 4. $\quad \boldsymbol{a}, \boldsymbol{b}$, Power and ITPC comparisons for the average of channels Fz and Cz, between correct and error trials $(\boldsymbol{a})$, and post-correct and post-error trials $(\boldsymbol{b})$. Significant $(p<0.001)$ regions are outlined in black using the MATLAB contourf function.

distracting presence of error feedback. Additionally, several studies have found PES with intertrial intervals of several seconds (Hajcak et al., 2003; Marco-Pallarés et al., 2008; King et al., 2010; Danielmeier et al., 2011), precluding a contribution of a transient disturbance of phase entrainment. However, these studies measured either $\mathrm{PES}_{\text {traditional, }}$ which is confounded by global fluctuations in motivation and task performance (Dutilh et al., 2012b), which are often present in tasks with long RSIs; or they used $\mathrm{PES}_{\text {robust }}$ but did not control for differences in trial type (e.g., congruent vs incongruent) between pre-error and posterror trials-a plausible source of confound (Steinhauser and Yeung, 2012). Therefore, it is uncertain whether PES truly occurred in these studies. Nonetheless, together, the literature suggests that in contrast to the intermediate RSI effects discussed in the previous paragraph, at longer RSIs PES is mainly determined by a timeconsuming strategic change in speed-accuracy tradeoff. This notion is corroborated by the finding that post-error accuracy is increased following errors only at longer RSIs (Jentzsch and Dudschig, 2009).
An important question is what neural mechanism orchestrates the alignment of neuronal oscillations to environmental rhythmicity. The widespread cortical topography and slow temporal dynamics of the entrained oscillations observed here suggest a possible neuromodulatory involvement. Accordingly, the norepinephrine-producing neurons of the locus ceruleus (LC) are phase locked in firing to ongoing cortical slow-wave oscillations during sleep (Eschenko et al., 2012; Sara and Bouret, 2012). Specifically, neurons of the prefrontal cortex and the LC fire in phasic opposition, suggesting a mutual excitatory drive. Additionally, one concurrent EEG-fMRI study has provided tentative evidence of temporal alignment of human LC activity with slowwave oscillations (Dang-Vu et al., 2008). These findings have led to the suggestion that the LC facilitates transitions from the down state to the up state of slow cortical oscillations (Eschenko et al., 2012; Sara and Bouret, 2012), which would provide a plausible mechanism for temporal alignment of slow oscillations with environmental rhythmicity. In line with the concept of an orienting response, phasic norepinephrine release has also been proposed 
to act as a neural interrupt signal, whereby unexpected events (e.g., errors) lead to a reset and reorganization in target neuronal networks, and subsequent behavioral adaptation (Bouret and Sara, 2005; Dayan and Yu, 2006). Interestingly, several proposals have linked PES to noradrenergic activity (Cohen et al., 2000; Núñez Castellar et al., 2010), and preliminary evidence suggests that PES is partly determined by a genetic marker of norepinephrine synthesis (Colzato et al., 2013). Thus, it is possible that the noradrenergic system is involved in the entrainment of cortical oscillations, and that this entrainment and consequent mode of behavioral responding are disrupted by an error-evoked orienting response (or interrupt signal) in the noradrenergic system (Nieuwenhuis et al., 2010; Núñez Castellar et al., 2010; Ullsperger et al., 2010).

A remaining question is whether our findings will generalize to other task designs. Here, phase entrainment followed the lowfrequency stimulus presentation rhythm. However, phase entrainment does not always occur in the lower frequency range (Maltseva et al., 2000), raising the question of whether an errorinduced disturbance might also occur in higher frequencies. Indeed, because we used only one stimulus presentation frequency, it is conceivable that the error-induced disturbance in phase angle occurred independent of endogenous entrainment. This possibility seems unlikely given that the choice of stimulus presentation rhythm was motivated by earlier work on entrainment (Stefanics et al., 2010; Lakatos et al., 2013) rather than a specific hypothesis about the involvement of $0.76 \mathrm{~Hz}$ oscillations in error processing. Furthermore, others have shown attentionrelated phase shifts of entrained oscillations in substantially higher frequencies (Lakatos et al., 2008; Besle et al., 2011). Nevertheless, the specificity of the error-related disturbance in phase angle to the task frequency warrants further investigation using multiple stimulus presentation rhythms.

Furthermore, in our experiment the task rhythm was determined mainly by the highly predictable SOA, whereas in many other studies of PES the RSI instead of the SOA is the predictable time interval. So, an interesting question is whether in these studies PES is also related to a disturbance of phase entrainment. Previous studies have shown that participants can use various temporal cues to facilitate entrainment (Stefanics et al., 2010). However, it remains to be investigated whether response time can function as a temporal cue to entrain oscillations to subsequent stimulus onset. Alternatively, to the extent that variability in RTs is modest and the RSI and other intervals are fixed, the SOA will be relatively constant across trials. The entrainment in the current study was robust to a certain amount of temporal variability (SOA, 1200-1500 ms), suggesting that, even in tasks in which RSI is the predictable factor, neuronal oscillations may entrain to the SOA.

In conclusion, our brain exploits the innate periodicity in the environment by means of oscillatory entrainment. We have shown that when an error is made, a temporary perturbation occurs such that the entrained oscillations are "out of sync" with the current task, the degree of which predicts the magnitude of slowing of the subsequent behavioral response.

\section{References}

Berens P (2009) CircStat: a MATLAB toolbox for circurlar statistics. J Stat Softw 31:1-21.

Besle J, Schevon CA, Mehta AD, Lakatos P, Goodman RR, McKhann GM, Emerson RG, Schroeder CE (2011) Tuning of the human neocortex to the temporal dynamics of attended events. J Neurosci 31:3176-3185. CrossRef Medline

Botvinick MM, Braver TS, Barch DM, Carter CS, Cohen JD (2001) Conflict monitoring and cognitive control. Psychol Rev 108:624-652. CrossRef Medline

Bouret S, Sara SJ (2005) Network reset: a simplified overarching theory of locus coeruleus noradrenaline function. Trends Neurosci 28:574-582. CrossRef Medline

Cavanagh JF, Cohen MX, Allen JJ (2009) Prelude to and resolution of an error: EEG phase synchrony reveals cognitive control dynamics during action monitoring. J Neurosci 29:98-105. CrossRef Medline

Cohen JD, Botvinick M, Carter CS (2000) Anterior cingulate and prefrontal cortes: who's in control? Nat Neurosci 3:421-423. CrossRef Medline

Cohen MX (2014) Analyzing neural time series data: theory and practice. Cambridge, MA: MIT.

Cohen MX, van Gaal S, Ridderinkhof KR, Lamme VA (2009) Unconscious errors enhance prefrontal-occipital oscillatory synchrony. Front Hum Neurosci 3:54. CrossRef Medline

Cohen MX, Bour L, Mantione M, Figee M, Vink M, Tijssen MA, van Rootselaar AF, van den Munckhof P, Schuurman PR, Denys D (2012) Topdown-directed synchrony from medial frontal cortex to nucleus accumbens during reward anticipation. Hum Brain Mapp 33:246-252. CrossRef Medline

Colzato LS, de Rover M, van den Wildenberg WP, Nieuwenhuis S (2013) Genetic marker of norepinephrine synthesis predicts individual differences in post-error slowing: a pilot study. Neuropsychologia 51:26002604. CrossRef Medline

Cravo AM, Rohenkohl G, Wyart V, Nobre AC (2013) Temporal expectation enhances contrast sensitivity by phase entrainment of low-frequency oscillations in visual cortex. J Neurosci 33:4002-4010. CrossRef Medline

Dang-Vu TT, Schabus M, Desseilles M, Albouy G, Boly M, Darsaud A, Gais S, Rauchs G, Sterpenich V, Vandewalle G, Carrier J, Moonen G, Balteau E, Degueldre C, Luxen A, Phillips C, Maquet P (2008) Spontaneous neural activity during human slow wave sleep. Proc Natl Acad Sci U S A 105: 15160-15165. CrossRef Medline

Danielmeier C, Ullsperger M (2011) Post-error adjustments. Front Psychol 2:233. CrossRef Medline

Danielmeier C, Eichele T, Forstmann BU, Tittgemeyer M, Ullsperger M (2011) Posterior medial frontal cortex activity predicts post-error adaptations in task-related visual and motor areas. J Neurosci 31:1780-1789. CrossRef Medline

Dayan P, Yu AJ (2006) Phasic norepinephrine: a neural interrupt signal for unexpected events. Network 17:335-350. CrossRef Medline

Delorme A, Makeig S (2004) EEGLAB: an open source toolbox for analysis of single-trial EEG dynamics including independent component analysis. J Neurosci Methods 134:9-21. CrossRef Medline

Donner TH, Siegel M (2011) A framework for local cortical oscillation patterns. Trends Cogn Sci 15:191-199. CrossRef Medline

Dudschig C, Jentzsch I (2009) Speeding before and slowing after errors: is it all just strategy? Brain Res 1296:56-62. CrossRef Medline

Dutilh G, Vandekerckhove J, Forstmann BU, Keuleers E, Brysbaert M, Wagenmakers EJ (2012a) Testing theories of post-error slowing. Atten Percept Psychophys 74:454-465. CrossRef Medline

Dutilh G, van Ravenzwaaij D, Nieuwenhuis S, van der Maas HLJ, Forstmann BU, Wagenmakers E-J (2012b) How to measure post-error slowing: a confound and a simple solution. J Math Psychol 56:208-216. CrossRef

Eichele H, Juvodden HT, Ullsperger M, Eichele T (2010) Mal-adaptation of event-related EEG responses preceding performance errors. Front Hum Neurosci 4:65. CrossRef Medline

Endrass T, Franke C, Kathmann N (2005) Error awareness in a saccade countermanding task. J Psychophysiol 19:275-280. CrossRef

Eriksen BA, Eriksen CW (1974) Effects of noise letters upon the identification of a target letter in a nonsearch task. Percept Psychophys 16:143-149. CrossRef

Eschenko O, Magri C, Panzeri S, Sara SJ (2012) Noradrenergic neurons of the locus coeruleus are phase locked to cortical up-down states during sleep. Cereb Cortex 22:426-435. CrossRef Medline

Fisher N (1993) Statistical analysis of circular data. Cambridge, UK Cambridge UP.

Gehring WJ, Fencsik DE (2001) Functions of the medial frontal cortex in the processing of conflict and errors. J Neurosci 21:9430-9437. Medline

Hajcak G, McDonald N, Simons RF (2003) To err is autonomic: errorrelated brain potentials, ANS activity, and post-error compensatory behavior. Psychophysiology 40:895-903. CrossRef Medline

Henry MJ, Obleser J (2012) Frequency modulation entrains slow neural 
oscillations and optimizes human listening behavior. Proc Natl Acad Sci U S A 109:20095-20100. CrossRef Medline

Hughes G, Yeung N (2011) Dissociable correlates of response conflict and error awareness in error-related brain activity. Neuropsychologia 49:405415. CrossRef Medline

Jentzsch I, Dudschig C (2009) Why do we slow down after an error? Mechanisms underlying the effects of posterror slowing. Q J Exp Psychol (Hove) 62:209-218. CrossRef Medline

King JA, Korb FM, von Cramon DY, Ullsperger M (2010) Post-error behavioral adjustments are facilitated by activation and suppression of taskrelevant and task-irrelevant information processing. J Neurosci 30: 12759-12769. CrossRef Medline

Lakatos P, Karmos G, Mehta AD, Ulbert I, Schroeder CE (2008) Entrainment of neuronal oscillations as a mechanism of attentional selection. Science 320:110-113. CrossRef Medline

Lakatos P, Schroeder CE, Leitman DI, Javitt DC (2013) Predictive suppression of cortical excitability and its deficit in schizophrenia. J Neurosci 33:11692-11702. CrossRef Medline

Laming D (1979) Choice reaction performance following an error. Acta Psychol (Amst) 43:199-224. CrossRef

Large EW, Jones MR (1999) The dynamics of attending: how people track time-varying events. Psychol Rev 106:119-159. CrossRef

Maltseva I, Giessler HG, Başar E (2000) Alpha oscillations as an indicator of dynamic memory operations - anticipation of omitted stimuli. Int J Psychophysiol 36:185-197. CrossRef Medline

Marco-Pallarés J, Camara E, Münte TF, Rodríguez-Fornells A (2008) Neural mechanisms underlying adaptive actions after slips. J Cogn Neurosci 20:1595-1610. CrossRef Medline

Maris E, Oostenveld R (2007) Nonparametric statistical testing of EEG- and MEG-data. J Neurosci Methods 164:177-190. CrossRef Medline

Murphy PR, Robertson IH, Allen D, Hester R, O'Connell RG (2012) An electrophysiological signal that precisely tracks the emergence of error awareness. Front Hum Neurosci 6:65. CrossRef Medline

Narayanan NS, Cavanagh JF, Frank MJ, Laubach M (2013) Common medial frontal mechanisms of adaptive control in humans and rodents. Nat Neurosci 16:1888-1895. CrossRef Medline

Ng BS, Schroeder T, Kayser C (2012) A precluding but not ensuring role of entrained low-frequency oscillations for auditory perception. J Neurosci 32:12268-12276. CrossRef Medline

Nieuwenhuis S, De Geus EJ, Aston-Jones G (2010) The anatomical and functional relationship between the $\mathrm{P} 3$ and autonomic components of the orienting response. Psychophysiology. Advance online publication. Retrieved July 14, 2014. doi:10.1111/j.1469-8986.2010.01057.x. CrossRef Medline

Notebaert W, Houtman F, Opstal FV, Gevers W, Fias W, Verguts T (2009)
Post-error slowing: an orienting account. Cognition 111:275-279. CrossRef Medline

Núñez Castellar E, Kühn S, Fias W, Notebaert W (2010) Outcome expectancy and not accuracy determines posterror slowing: ERP support. Cogn Affect Behav Neurosci 10:270-278. CrossRef Medline

Rabbitt PM (1966) Errors and error correction in choice-response tasks. J Exp Psychol 71:264-272. CrossRef Medline

Ridderinkhof KR (2002) Micro- and macro-adjustments of task set: activation and suppression in conflict tasks. Psychol Res 66:312-323. CrossRef Medline

Saleh M, Reimer J, Penn R, Ojakangas CL, Hatsopoulos NG (2010) Fast and slow oscillations in human primary motor cortex predict oncoming behaviorally relevant cues. Neuron 65:461-471. CrossRef Medline

Sara SJ, Bouret S (2012) Orienting and reorienting: the locus coeruleus mediates cognition through arousal. Neuron 76:130-141. CrossRef Medline

Schroeder CE, Lakatos P (2009) Low-frequency neuronal oscillations as instruments of sensory selection. Trends Neurosci 32:9-18. CrossRef Medline

Stefanics G, Hangya B, Hernádi I, Winkler I, Lakatos P, Ulbert I (2010) Phase entrainment of human delta oscillations can mediate the effects of expectation on reaction speed. J Neurosci 30:13578-13585. CrossRef Medline

Steinborn MB, Flehmig HC, Bratzke D, Schroter H (2012) Error reactivity in self-paced performance: highly-accurate individuals exhibit largest post-error slowing. Q J Exp Psychol (Hove) 65:624-631. CrossRef

Steinhauser M, Yeung N (2012) Error awareness as evidence accumulation: effects of speed-accuracy trade-off on error signaling. Front Hum Neurosci 6:240. CrossRef Medline

Tallon-Baudry C, Bertrand O (1999) Oscillatory gamma activity in humans and its role in object representation. Trends Cogn Sci 3:151-162. CrossRef Medline

Ullsperger M, Harsay HA, Wessel JR, Ridderinkhof KR (2010) Conscious perception of errors and its relation to the anterior insula. Brain Struct Funct 214:629-643. CrossRef Medline

Wang XJ (2010) Neurophysiological and computational principles of cortical rhythms in cognition. Physiol Rev 90:1195-1268. CrossRef Medline

White CN, Ratcliff R, Vasey MW, McKoon G (2010) Using diffusion models to understand clinical disorders. J Math Psychol 54:39-52. CrossRef Medline

Wyart V, de Gardelle V, Scholl J, Summerfield C (2012) Rhythmic fluctuations in evidence accumulation during decision making in the human brain. Neuron 76:847-858. CrossRef Medline

Zar JH (1999) Biostatistical analysis, Ed5. Upper Saddle River, NJ: Prentice Hall. 